\title{
On zeros of regular orthogonal polynomials on the unit circle
}

\author{
by P. García Lázaro and F. Marcellán (Madrid)
}

\begin{abstract}
A new approach to the study of zeros of orthogonal polynomials with respect to an Hermitian and regular linear functional is presented. Some results concerning zeros of kernels are given.
\end{abstract}

1. Introduction. Zeros of orthogonal polynomials with respect to finite positive Borel measures whose support is an infinite subset of $\mathbb{R}$ play an important role in subjects ranging from mathematics to physics as:

- Numerical integration, mainly in the context of construction of Gaussian quadratures.

- Lanczos method and Jacobi matrices whose eigenvalues are zeros of an orthogonal polynomial sequence defined by a three-term recurrence relation.

- Quantum mechanics where in many cases, the zeros of orthogonal polynomials are the zeros of the Schrödinger equation solutions in both the position and the momentum spaces.

- Many body problems in quantum physics in which the zeros of orthogonal polynomials are the energy levels of the physical system.

- Padé approximation where they are the poles of the $n$th approximant.

Some well known properties of these zeros are:

a) Multiplicity equal to one.

b) They are located in the convex hull of the support of the measure.

c) The so called interlacing property, that is, if we denote by $\left(x_{n, i}\right)_{i=1}^{n}$ the zeros of $P_{n}$ taken in increasing order then $x_{n, i}<x_{n-1, i}<x_{n, i+1}$ for $i=1,2, \ldots, n-1$.

1991 Mathematics Subject Classification: Primary 42C05.

Key words and phrases: zeros, orthogonal polynomials, Toeplitz matrices, regular functional.

Research of the second author partially supported by a Proyecto Concertado of Comisión Interministerial de Ciencia y Tecnología (CICYT) number PB89-0181-C02-01. 
On the contrary, there are very few results about the distribution of zeros in the case of orthogonal polynomials with respect to finite positive Borel measures when the support is an infinite subset of $T=\{z \in \mathbb{C}:|z|=1\}$. This is so despite the fact that they are quite important in subjects like:

1. Stability theory in discrete linear systems. In particular, the celebrated Schur-Cohn algorithm.

2. Numerical integration on the unit circle.

3. Problems on trigonometric moments.

In this paper we deal with the zeros of orthogonal polynomials $\left(\Phi_{n}(z)\right)_{n>0}$ with respect to regular functionals, which are the natural generalization of the positive definite case. In Section 2 some basic definitions and ideas are introduced. In particular, the Szegö recurrence relations and the ChristoffelDarboux formula are crucial when proving some propositions in Section 3. In Section 3 we analyze the distribution of zeros of sequences of orthogonal polynomials associated with a regular functional.

The basic results are:

1. There are no zeros of the sequence of orthogonal polynomials $\left(\Phi_{n}(z)\right)_{n \geq 0}$ on the unit circle and if $\Phi_{n}(\alpha)=0$ then $\Phi_{n}\left(\overline{\alpha^{-1}}\right) \neq 0$. Moreover, if $\Phi_{n}(0) \neq 0$ for some $n \geq 0$ then $\Phi_{n}(z)$ and $\Phi_{n-1}(z)$ do not have any common root.

2. Suppose $\Phi_{n}(z)$ has $p$ zeros inside and $q$ zeros outside $T$. Then:

2a. $\left|\Phi_{n+1}(0)\right|<1$ implies that $\Phi_{n+1}(z)$ has $p+1$ zeros inside and $q$ zeros outside $T$.

2b. If $\left|\Phi_{n+1}(0)\right|>1$ then $\Phi_{n+1}(z)$ has $q$ zeros inside and $p+1$ zeros outside $T$.

By means of the Szegö-Schur parameters $\Phi_{n}(0)$, we give an analytic proof of the above mentioned result. It is essentially different from those given by M. G. Krein (see [8]) and R. L. Ellis et al. (see [1]), which use algebraic techniques.

In Section 3 we also study the zeros of the kernels associated with a regular functional pointing out the difference with the positive definite case.

2. Regular functionals. Let

$$
M=\left[c_{n}\right]_{n \geq 0}=\left[\begin{array}{ccccc}
c_{0} & c_{1} & \ldots & c_{n} & \ldots \\
\bar{c}_{1} & c_{0} & \ldots & c_{n-1} & \ldots \\
\vdots & \vdots & \ddots & \vdots & \vdots \\
\bar{c}_{n} & \bar{c}_{n-1} & \ldots & c_{0} & \ldots \\
\vdots & \vdots & \ddots & \vdots & \vdots
\end{array}\right]
$$

be an infinite Hermitian Toeplitz matrix. 
In the space $\Lambda=L\left[z^{n}\right]_{n \in \mathbb{Z}}$ of Laurent polynomials with complex coefficients, we consider a functional $u$ associated with $M$ such that

$$
c_{n}=\left\langle u, z^{n}\right\rangle \quad \text { and } \quad \bar{c}_{n}=\left\langle u, z^{-n}\right\rangle \quad \forall n \in \mathbb{N} .
$$

Then $u$ defines a bilinear form in the space $\mathbb{P}$ of polynomials with complex coefficients by means of

$$
\varphi_{u}(\Phi(z), \Psi(z))=\left\langle u, \Phi(z) \overline{\Psi\left(\overline{z^{-1}}\right)}\right\rangle,
$$

so that the Gram matrix corresponding to the canonical basis $\left(z^{n}\right)_{n \in \mathbb{N}}$ is $M$.

Let $\mathbb{P}_{n}$ be the linear subspace of $\mathbb{P}$ of polynomials with degree less than or equal to $n, M_{n}$ the principal submatrices of $M$ and $\Delta_{n}=\operatorname{det} M_{n}, n \geq 0$.

The linear functional $u$ or, equivalently, the bilinear form $\varphi_{u}$ is regular (see [10]) or quasidefinite (see [7]) if $\forall n \geq 0, \Delta_{n} \neq 0$. We say that $u$ is positive definite if $\forall n \geq 0, \Delta_{n}>0$.

If $u$ is a positive definite functional it is well known that there is a finite and positive Borel measure $\mu$ with infinite support on $T$ such that

$$
\langle u, \Phi(z)\rangle=\int_{T} \Phi(z) d \mu(z)
$$

with $T=\{z \in \mathbb{C}:|z|=1\}$. The result does not hold if $u$ is just a regular functional and of course it is easy to find functionals like $\delta_{a}: \mathbb{P} \rightarrow \mathbb{P},\left\langle\delta_{a}\right.$, $p(z)\rangle=p(a)$ with $|a|=1$, that are not regular (see [4]).

Assuming regularity, the Gram-Schmidt orthonormalization procedure allows us to define a family of orthogonal polynomials $\left(\Phi_{n}(z)\right)_{n \geq 0}$ by

$$
\Phi_{n}(z)=A_{n} \operatorname{det}\left[\begin{array}{cccc}
c_{0} & c_{1} & \ldots & c_{n} \\
\bar{c}_{1} & c_{0} & \ldots & c_{n-1} \\
\vdots & \vdots & \ddots & \vdots \\
\bar{c}_{n-1} & \bar{c}_{n-2} & \ldots & c_{1} \\
1 & z & \ldots & z_{n}
\end{array}\right], \quad A_{n} \neq 0
$$

such that

$$
\left\langle u, \Phi_{n}(z) \overline{\Phi_{m}\left(\overline{z^{-1}}\right)}\right\rangle=L_{n} \delta_{n, m} \quad \text { with } L_{n} \neq 0 \quad \forall n, m \geq 0 .
$$

If $\forall n \geq 0, \Phi_{n}(z)$ is monic, then we call $\left(\Phi_{n}(z)\right)_{n \geq 0}$ a monic orthogonal polynomial sequence (M.O.P.S.).

By using algebraic techniques in $M_{n+1}$ it can be proved that (see [10])

$$
\frac{e_{n+1}}{e_{n}}=1-\left|\Phi_{n+1}(0)\right|^{2}, \quad n \geq 0,
$$

with $e_{n}=\Delta_{n} / \Delta_{n-1}, n \geq 0$, and the convention $\Delta_{-1}=1$. Then from the fact that $u$ is regular we get $\left|\Phi_{n}(0)\right| \neq 1, n \geq 1$. In particular, if the functional is positive definite, it is well known that $\left|\Phi_{n}(0)\right|<1$ (see [10]). 
Let $\Phi(z)$ be a polynomial of degree $n$. We say that $\Phi^{*}(z)$ is the reciprocal polynomial of $\Phi(z)$ if $\Phi^{*}(z)=z^{n} \overline{\Phi\left(\overline{z^{-1}}\right)}$. One of the points of interest concerning orthogonal polynomials on the unit circle in the positive definite case is the study of Fourier series for $L^{2}(\mu)$ functions. To be more specific, the $n$th Fourier partial sum with respect to the given family of orthogonal polynomials can be written in terms of the $n$th kernel $K_{n}(z, y)$ as

$$
S_{n}(z, f)=\int_{T} K_{n}(z, y) f(y) d \mu, \quad K_{n}(z, y)=\sum_{k=0}^{n} \frac{\Phi_{k}(z) \overline{\Phi_{k}(y)}}{e_{k}} .
$$

$\Phi_{n}^{*}(z)$ is related to $K_{n}(z, y)$ by means of

$$
\Phi_{n}^{*}(z)=e_{n} K_{n}(z, 0) .
$$

It is known that the shift operator in $L^{2}(\mu)$ is unitary with respect to the scalar product induced by the measure. In the regular case the property is still valid and it proves useful when getting the so called recurrence relations satisfied by the M.O.P.S.: for all $n \geq 0$,

$$
\begin{aligned}
& \Phi_{n+1}(z)=z \Phi_{n}(z)+\Phi_{n+1}(0) \Phi_{n}^{*}(z), \\
& \Phi_{n+1}^{*}(z)=\Phi_{n}^{*}(z)+\overline{\Phi_{n+1}(0)} z \Phi_{n}(z), \\
& \Phi_{n+1}(z)=\frac{e_{n+1}}{e_{n}} z \Phi_{n}(z)+\Phi_{n+1}(0) \Phi_{n+1}^{*}(z), \\
& \Phi_{n+1}^{*}(z)=\frac{e_{n+1}}{e_{n}} \Phi_{n}^{*}(z)+\overline{\Phi_{n+1}(0)} \Phi_{n+1}(z) .
\end{aligned}
$$

These four equivalent recurrence formulas were obtained by Szegö (see [12]) in the positive definite case and by Geronimus in the regular case (see [5]).

From (3), regular functionals can be characterized in the following way.

Proposition 2.1. If $\left(\Phi_{n}(z)\right)_{n \geq 0}$ is a sequence of monic polynomials such that $\forall n \geq 0, \operatorname{deg} \Phi_{n}(z)=n$ and

$$
\forall n \geq 1, \quad \Phi_{n}(z)=z \Phi_{n-1}(z)+\Phi_{n}(0) \Phi_{n-1}^{*}(z),
$$

then there is a (Hermitian) linear functional $u$ in $\Lambda$ such that $\left(\Phi_{n}(z)\right)_{n \geq 0}$ is a M.O.P.S. with respect to $u$ if $\forall n \geq 1,\left|\Phi_{n}(0)\right| \neq 1$ (see [10]).

The result is a generalization of the Favard theorem in the positive definite case for $T$ (see [2]).

Also from (3) we can obtain explicitly the $n$th moment of the functional $u$ in terms of the preceding moments and the coefficients of the $n$th orthogonal polynomial associated with $u$. In fact, from (3),

$$
\left\langle\Phi_{n}(z), 1\right\rangle=0 \quad \text { and then } \quad c_{n}=-\sum_{k=0}^{n-1} a_{n, k} c_{k}
$$


where the $a_{n, k}$ are the coefficients of the polynomial $\Phi_{n}(z)$ and $c_{k}$ the moments of the functional $u$.

A parallel procedure in the positive definite case is given by the so called Gohberg-Semencul formula (see [11]). If $\left(a_{k}\right)_{k=0}^{n}$ are the coefficients of the $n$th orthogonal polynomial associated with $u$, the matrix

$$
\begin{aligned}
{\left[\begin{array}{cccc}
\bar{a}_{n} & 0 & \ldots & 0 \\
\bar{a}_{n-1} & \bar{a}_{n} & \ldots & 0 \\
\vdots & \vdots & \ddots & \vdots \\
\bar{a}_{1} & \bar{a}_{2} & \ldots & \bar{a}_{n}
\end{array}\right]\left[\begin{array}{cccc}
a_{n} & a_{n-1} & \ldots & a_{1} \\
0 & a_{n} & \ldots & a_{2} \\
\vdots & \vdots & \ddots & \vdots \\
0 & 0 & \ldots & a_{n}
\end{array}\right] } \\
-\left[\begin{array}{cccc}
a_{0} & 0 & \ldots & 0 \\
a_{1} & a_{0} & \ldots & 0 \\
\vdots & \vdots & \ddots & \vdots \\
a_{n-1} & a_{n-2} & \ldots & a_{0}
\end{array}\right]\left[\begin{array}{cccc}
\bar{a}_{0} & \bar{a}_{1} & \ldots & \bar{a}_{n-1} \\
0 & \bar{a}_{0} & \ldots & \bar{a}_{n-2} \\
\vdots & \vdots & \ddots & \vdots \\
0 & 0 & \ldots & \bar{a}_{0}
\end{array}\right]
\end{aligned}
$$

is the inverse of the $(n+1)$ th Toeplitz matrix associated with the scalar product induced by $u$.

The M.O.P.S. $\left(\Phi_{n}(z)\right)_{n \geq 0}$ satisfies a three-term recurrence relation which is more complex than the above mentioned ones:

$$
\begin{aligned}
\widetilde{a}_{n} \Phi_{n+1}(z)=\left(z \widetilde{a}_{n}+a_{n+1}\right) \Phi_{n}(z)-\widetilde{a}_{n+1}\left(1-\left|\widetilde{a}_{n}\right|^{2}\right) & z \Phi_{n-1}(z), \\
n & \geq 1, \widetilde{a}_{n}=\Phi_{n}(0) .
\end{aligned}
$$

With the help of the recurrence formulas it is possible to deduce a representation for the kernels which is analogous to the Christoffel-Darboux formula for orthogonal polynomial sequences on the real line. Such a formula can be obtained through the Bézoutian associated with $\Phi_{n}(z)$ and $\Phi_{n}^{*}(z)$ by means of

$$
e_{n+1}(z-y) K_{n}^{*}(z, y)=\Phi_{n+1}^{*}(y) \Phi_{n+1}(z)-\Phi_{n+1}(y) \Phi_{n+1}^{*}(z)
$$

for $n \geq 0$ where

$$
K_{n}^{*}(z, y)=\sum_{k=0}^{n} \frac{1}{e_{k}} z^{n-k} \Phi_{k}^{*}(z) \Phi_{k}(y),
$$

and the latter can be obtained directly from the following representation for the $n$th kernels:

$$
e_{n+1}(1-z \bar{y}) K_{n}(z, y)=\Phi_{n+1}^{*}(z) \overline{\Phi_{n+1}^{*}(y)}-\Phi_{n+1}(z) \overline{\Phi_{n+1}(y)} .
$$

3. Zeros. Let $u$ be a positive definite linear functional. Then (see [12]) the zeros of the M.O.P.S. associated with $u$ are inside the unit disc.

Proposition 3.1. Let $\left(\Phi_{n}(z)\right)_{n \geq 0}$ be a M.O.P.S. with respect to the regular functional $u$ and $Z_{n}$ the set of zeros of $\Phi_{n}(z)$. Then

(i) If $\alpha \neq 0, \Phi_{n}(\alpha)=0 \Rightarrow \Phi_{n}\left(\overline{\alpha^{-1}}\right) \neq 0$. 
(ii) $\Phi_{n}(z)$ and $\Phi_{n}^{*}(z)$ do not have common roots.

(iii) $\Phi_{n}(\alpha)=0 \Rightarrow|\alpha| \neq 1$ and as a straightforward consequence: $\Phi_{n}^{*}(\alpha)=0 \Rightarrow|\alpha| \neq 1$.

(iv) Given two consecutive polynomials $\Phi_{n-1}(z)$ and $\Phi_{n}(z)$ we have

(a) If $\Phi_{n}(0)=0$ then $Z_{n}=Z_{n-1} \cup\{0\}$.

(b) If $\Phi_{n}(0) \neq 0$ then $Z_{n} \cap Z_{n-1}=\emptyset$.

Proof. (i) Suppose that $\Phi_{n}(\alpha)=\Phi_{n}\left(\overline{\alpha^{-1}}\right)=0$. Then, as $\Phi_{n}(\alpha)=0 \Leftrightarrow$ $\Phi_{n}^{*}\left(\overline{\alpha^{-1}}\right)=0$ we have $\Phi_{n}^{*}(\alpha)=\Phi_{n}^{*}\left(\overline{\alpha^{-1}}\right)=0$. (5) with $z=\alpha$ and $z=\overline{\alpha^{-1}}$ implies that $\Phi_{n-1}^{*}(\alpha)=\Phi_{n-1}^{*}\left(\overline{\alpha^{-1}}\right)=0$ and by iteration we deduce that $\alpha$ and $\overline{\alpha^{-1}}$ are roots of $\Phi_{1}(z)$. Then $\Phi_{1}(z)=z-\alpha$ and thus $\left|\Phi_{1}(0)\right|=1$, contrary to the hypothesis of the regularity of $u$.

(ii) $\Phi_{n}(\alpha)=\Phi_{n}^{*}(\alpha)=0$ implies $\Phi_{n}\left(\overline{\alpha^{-1}}\right)=0$, which contradicts (i).

(iii) This is a direct consequence of (ii).

(iv) (a) Straightforward from (iii).

(iv) (b) If there is $\alpha \neq 0 \in \mathbb{C}$ such that $\Phi_{n}(\alpha)=\Phi_{n-1}(\alpha)=0$, then writing, in (iii), $z=\alpha$ we get $\Phi_{n}(0) \Phi_{n}^{*}(\alpha)=0$. Thus, $\Phi_{n}^{*}(\alpha)=0$, which contradicts (ii).

Remark. An alternative way of proving (ii) goes as follows. If $\alpha$ and $\overline{\alpha^{-1}}$ are zeros of $\Phi_{n}(z)$ then $\Phi_{n}(z)=(z-\alpha) P_{n-1}(z)=\left(z-\overline{\alpha^{-1}}\right) Q_{n-1}(z)$ with $P_{n-1}, Q_{n-1} \in \mathbb{P}_{n-1}$ and

$$
\begin{aligned}
\left\langle u, P_{n-1}(z) \overline{P_{n-1}\left(\overline{z^{-1}}\right)}\right\rangle & =\left\langle u, \frac{z-\overline{\alpha^{-1}}}{z-\alpha} Q_{n-1}(z) \frac{1-\overline{\alpha^{-1}} z}{1-\bar{\alpha} z} \overline{Q_{n-1}\left(\overline{z^{-1}}\right)}\right\rangle \\
& =|\alpha|^{-2}\left\langle u, Q_{n-1}(z) \overline{\left.Q_{n-1}\left(\overline{z^{-1}}\right)\right\rangle} .\right.
\end{aligned}
$$

On the other hand,

$$
\begin{aligned}
\left\langle u, \Phi_{n}(z) \overline{\Phi_{n}\left(\overline{z^{-1}}\right)}\right\rangle & =\left(1-|\alpha|^{2}\right)\left\langle u, P_{n-1}(z) \overline{P_{n-1}\left(\overline{z^{-1}}\right)}\right\rangle \\
& =|\alpha|^{-2}\left(|\alpha|^{2}-1\right)\left\langle u, Q_{n-1}(z) \overline{Q_{n-1}\left(\overline{z^{-1}}\right)}\right\rangle .
\end{aligned}
$$

Thus, $\left\langle u, \Phi_{n}(z) \overline{\Phi_{n}\left(\overline{z^{-1}}\right)}\right\rangle=0$, contrary to the regularity of the linear functional $u$.

Notice that in the first proof the existence of an orthogonal basis in $\mathbb{P}_{n}$ is needed, while in the second one we only require the existence of a polynomial of a given degree, orthogonal to all the preceding ones, and the following characterization:

$$
\left\langle u, \Phi_{n}(z) z^{-m}\right\rangle=L_{n} \delta_{n m} \quad \forall n, m \geq 0, m \leq n \text { and } L_{n} \neq 0 .
$$

In the sequel we study the properties of the zeros of the $n$th kernel $K_{n}(z, \alpha)$ getting more restrictive results than in the positive definite case. In the latter (see [3]): 
1) If $|\alpha| \neq 1$, the roots of $K_{n}(z, \alpha)$ are not on the unit circle, more precisely, if $|\alpha|>1$ the roots are located inside and if $|\alpha|<1$ outside the unit circle.

2) If $|\alpha|=1$ the roots of $K_{n}(z, \alpha)$ are on $T$.

Proposition 3.2. Let $\left(\Phi_{n}(z)\right)_{n \geq 0}$ be the M.O.P.S. associated with the regular functional $u$. For $\alpha \neq 0, \alpha \in \mathbb{C}$, we have:

(a) If $\left|\Phi_{n}(\alpha)\right| \neq\left|\Phi_{n}^{*}(\alpha)\right|$ and $K_{n-1}(\beta, \alpha)=0$ with $\beta \neq 0$ then $\beta \neq \alpha$, $|\beta| \neq 1$ and $K_{n-1}\left(\overline{\beta^{-1}}, \alpha\right) \neq 0$.

(b) If $\left|\Phi_{n}(\alpha)\right|=\left|\Phi_{n}^{*}(\alpha)\right|$ and $K_{n-1}(\beta, \alpha)=0$ with $\beta \neq 0$ then either $K_{n-1}\left(\overline{\beta^{-1}}, \alpha\right)=0$ or $\beta=\alpha$.

Proof. (a) From (7) it we get

$$
\Phi_{n}^{*}(\beta) \overline{\Phi_{n}^{*}(\alpha)}=\Phi_{n}(\beta) \overline{\Phi_{n}(\alpha)} .
$$

Thus $\beta \neq \alpha$ and, taking into account $\left|\Phi_{n}(\alpha)\right| \neq\left|\Phi_{n}^{*}(\alpha)\right|$, we have $|\alpha| \neq 1$. Now, either $\Phi_{n}(\alpha)=0$ or $\Phi_{n}(\alpha) \neq 0$.

If $\Phi_{n}(\alpha)=0$ then from $(8), \Phi_{n}^{*}(\beta) \overline{\Phi_{n}^{*}(\alpha)}=0$, and from Proposition 3.1(ii) we find that $\Phi_{n}^{*}(\beta)=0$. Then $|\beta| \neq 1$ and $\Phi_{n}^{*}\left(\overline{\beta^{-1}}\right) \neq 0$. Thus, taking into account that

$$
e_{n}\left(1-\overline{\alpha \beta^{-1}}\right) K_{n-1}\left(\overline{\beta^{-1}}, \alpha\right)=\Phi_{n}^{*}\left(\overline{\beta^{-1}}\right) \overline{\Phi_{n}^{*}(\alpha)}
$$

with $\beta \neq 0$, we get $K_{n-1}\left(\overline{\beta^{-1}}, \alpha\right) \neq 0$ and $|\beta| \neq 1$.

If $\Phi_{n}(\alpha) \neq 0$ and $K_{n-1}(\beta, \alpha)=0$, then (7) yields

$$
\Phi_{n}(\beta)=\frac{\Phi_{n}^{*}(\beta) \overline{\Phi_{n}^{*}(\alpha)}}{\overline{\Phi_{n}(\alpha)}}
$$

and then $|\beta| \neq 1$ because if not we would have $\left|\Phi_{n}(\alpha)\right|=\left|\Phi_{n}^{*}(\alpha)\right|$. For $\beta \neq 0$,

$$
\begin{aligned}
e_{n}\left(1-\overline{\alpha \beta^{-1}}\right) K_{n-1}\left(\overline{\beta^{-1}}, \alpha\right) & =\Phi_{n}^{*}\left(\overline{\beta^{-1}}\right) \overline{\Phi_{n}^{*}(\alpha)}-\Phi_{n}\left(\overline{\beta^{-1}}\right) \overline{\Phi_{n}(\alpha)} \\
& =\left(\overline{\beta^{-1}}\right)^{n} \overline{\Phi_{n}(\beta)} \overline{\Phi_{n}^{*}(\alpha)}-\left(\overline{\beta^{-1}}\right)^{n} \overline{\Phi_{n}^{*}(\beta)} \overline{\Phi_{n}(\alpha)}
\end{aligned}
$$

and from (9),

$$
e_{n}\left(1-\overline{\alpha \beta^{-1}}\right) K_{n-1}\left(\overline{\beta^{-1}}, \alpha\right)=\left(\overline{\beta^{-1}}\right)^{n} \frac{\overline{\Phi_{n}^{*}(\beta)}}{\Phi_{n}(\alpha)}\left(\left|\Phi_{n}^{*}(\alpha)\right|^{2}-\left|\Phi_{n}(\alpha)\right|^{2}\right) .
$$

Thus, $K_{n-1}\left(\overline{\beta^{-1}}, \alpha\right) \neq 0$.

(b) If $\left|\Phi_{n}(\alpha)\right|=\left|\Phi_{n}^{*}(\alpha)\right|$ then $\Phi_{n}(\alpha) \neq 0$ and from (10) we get the result.

If $|z|=1$, it is clear that $\left|\Phi_{n}(z)\right|=\left|\Phi_{n}^{*}(z)\right|$ for a positive definite or regular functional $u$. For positive definite functionals and $\left|\Phi_{n}(z)\right|=\left|\Phi_{n}^{*}(z)\right|$, $\varphi(z)=\Phi_{n}(z) / \Phi_{n}^{*}(z)$ is an analytic function in $D=\{z \in \mathbb{C}:|z|<1\}$. By the maximum modulus theorem the maximum of $\varphi$ is on the boundary and so $|\varphi(z)|<1$ for $|z|<1$ and $|\varphi(z)|>1$ for $|z|>1$. 
For regular functionals $\varphi(z)$ is analytic neither inside nor outside the unit disc and then we can have $\left|\Phi_{n}(z)\right|=\left|\Phi_{n}^{*}(z)\right|$ with $|z|=1$. In fact, consider the M.O.P.S. defined by means of $\Phi_{1}(0)=-2$ and $\Phi_{2}(0)=0$. Then $\left|\Phi_{2}(z)\right|=\left|\Phi_{2}^{*}(z)\right|$ despite the fact that $|z|=1$ or $|z-2|^{2}=3$.

COROllary 3.3. For regular functionals we have:

(i) $\left|\Phi_{n}(\alpha)\right|=\left|\Phi_{n}^{*}(\alpha)\right| \Rightarrow K_{n-1}(0, \alpha) \neq 0$.

(ii) If $|\beta|=1$ and $K_{n-1}(\beta, \alpha)=0$, then

$$
\left|\Phi_{n}(\alpha)\right|=\left|\Phi_{n}^{*}(\alpha)\right| \quad \text { and } \quad|\alpha|\left|\Phi_{n-1}(\alpha)\right|=\left|\Phi_{n-1}^{*}(\alpha)\right| .
$$

Moreover,

$$
\left|\frac{\Phi_{n}^{*}(\alpha)}{\Phi_{n-1}^{*}(\alpha)}\right| \leq 1+\left|\Phi_{n}(0)\right| \quad \text { and } \quad\left|\frac{\Phi_{n}(\alpha)}{\Phi_{n-1}(\alpha)}\right| \leq|\alpha|\left(1+\left|\Phi_{n}(0)\right|\right) .
$$

Proof. (i) Suppose that $K_{n-1}(0, \alpha)=0$. By $(2), \Phi_{n-1}^{*}(\alpha)=0$ and then $\alpha \neq 0$. On the other hand, from (3) and (4),

$$
\Phi_{n}^{*}(\alpha)=\overline{\Phi_{n}(0)} \alpha \Phi_{n-1}(\alpha), \quad \Phi_{n}(\alpha)=\alpha \Phi_{n-1}(\alpha) .
$$

Imposing $\left|\Phi_{n}(\alpha)\right|=\left|\Phi_{n}^{*}(\alpha)\right|$ and taking into account that $\Phi_{n-1}(\alpha) \neq 0$, from (12) we obtain $|\alpha|=\left|\Phi_{n}(0)\right||\alpha|$ and so $\alpha=0$, and the result follows.

(ii) If $|\beta|=1$ and $K_{n-1}(\beta, \alpha)=0$, from (7) we have

$$
\beta^{n} \overline{\Phi_{n}(\beta)} \overline{\Phi_{n}^{*}(\alpha)}=\Phi_{n}(\beta) \overline{\Phi_{n}(\alpha)} \text {. }
$$

Therefore, $\left|\Phi_{n}(\alpha)\right|=\left|\Phi_{n}^{*}(\alpha)\right|$. It is known (see [10]) that

$$
e_{n}(1-z \bar{y}) K_{n}(z, y)=\Phi_{n}^{*}(z) \overline{\Phi_{n}^{*}(y)}-z \bar{y} \Phi_{n}(z) \overline{\Phi_{n}(y)}
$$

which yields

$$
\beta^{n-2} \overline{\Phi_{n-1}(\beta)} \overline{\Phi_{n-1}^{*}(\alpha)}=\bar{\alpha} \Phi_{n-1}(\beta) \overline{\Phi_{n-1}(\alpha)}
$$

Thus

$$
\left|\Phi_{n-1}^{*}(\alpha)\right|=|\alpha|\left|\Phi_{n-1}(\alpha)\right|
$$

From $\alpha \Phi_{n-1}(\alpha)=\Phi_{n}(\alpha)-\Phi_{n}(0) \Phi_{n-1}^{*}(\alpha)$, we obtain

$$
\left|\frac{\Phi_{n}(\alpha)}{\Phi_{n-1}^{*}(\alpha)}-\Phi_{n}(0)\right|=1
$$

and hence

$$
\left|\frac{\Phi_{n}(\alpha)}{\Phi_{n-1}^{*}(\alpha)}\right| \leq 1+\left|\Phi_{n}(0)\right|
$$

From (11) and (4),

$$
\frac{\left|\Phi_{n}(\alpha)\right|}{\left|\Phi_{n-1}(\alpha)\right|}=\frac{|\alpha|\left|\Phi_{n}^{*}(\alpha)\right|}{\left|\Phi_{n-1}^{*}(\alpha)\right|}=|\alpha|\left|1+\alpha \overline{\Phi_{n}(0)} \frac{\Phi_{n-1}(\alpha)}{\Phi_{n-1}^{*}(\alpha)}\right|,
$$


and finally

$$
\left|\frac{\Phi_{n}(\alpha)}{\Phi_{n-1}(\alpha)}\right| \leq|\alpha|\left(1+\left|\Phi_{n}(0)\right|\right) .
$$

Re mark. Let $\alpha \in \mathbb{C}$ be a zero of the $n$th orthogonal polynomial $\Phi_{n}(z)$. Up to now we have seen that $|\alpha| \neq 1$ and, moreover, if $\Phi_{n}(\alpha)=0$ then $\Phi_{n}\left(\overline{\alpha^{-1}}\right) \neq 0$. In what follows we are going to prove that from the number of zeros of $\Phi_{n}(z)$ inside the unit circle it is possible to obtain the number of zeros inside the unit circle (and then outside as well) for the next polynomial $\Phi_{n+1}(z)$.

Proposition 3.4. Let $\left(\Phi_{n}(z)\right)_{n \geq 0}$ be a M.O.P.S. with respect to the regular functional $u$. If $\Phi_{n}(z)$ has $p$ zeros inside and $q$ zeros outside $T$, then we have:

(i) If $\left|\Phi_{n+1}(0)\right|<1$ then $\Phi_{n+1}(z)$ has $p+1$ zeros inside and $q$ zeros outside $T$.

(ii) If $\left|\Phi_{n+1}(0)\right|>1$ then $\Phi_{n+1}(z)$ has $q$ zeros inside and $p+1$ zeros outside $T$.

Proof. (i) First of all, we write $\Phi_{n}(z)$ as

$$
\Phi_{n}(z)=I_{p}(z) E_{q}(z) \quad \text { with } p+q=n
$$

where $I_{p}(z)$ is a polynomial of degree $p$ with $p$ zeros inside $T$, and $E_{q}(z)$ a polynomial of degree $q$ with $q$ zeros outside $T$.

Next, we prove the existence of two holomorphic functions

$$
f(z)=\frac{\Phi_{n+1}^{*}(z)}{I_{p}^{*}(z)}, \quad g(z)=E_{q}^{*}(z),
$$

to which Rouché's theorem can be applied.

For a polynomial of degree $n$ we consider the operator $*: \mathbb{P} \rightarrow \mathbb{P}$ defined as $\Phi^{*}(z)=z^{n} \overline{\Phi\left(\overline{z^{-1}}\right)}$. Applying $*$ to (13) gives

$$
\Phi_{n}^{*}(z)=I_{p}^{*}(z) E_{q}^{*}(z) .
$$

If $z \in \mathbb{C}$ with $|z|=1$, then $\left|\Phi_{n}(z)\right|=\left|\Phi_{n}^{*}(z)\right|$ and from (4) and (13) we have

$$
\begin{aligned}
\left|\frac{\Phi_{n+1}^{*}(z)-\Phi_{n}^{*}(z)}{I_{p}^{*}(z)}\right| & =\left|\frac{\overline{\Phi_{n+1}(0)} z \Phi_{n}(z)}{I_{p}^{*}(z)}\right|=\left|\frac{I_{p}(z) E_{q}(z)}{I_{p}^{*}(z)}\right|\left|\Phi_{n+1}(0)\right| \\
& =\left|\Phi_{n+1}(0)\right|\left|E_{q}^{*}(z)\right| .
\end{aligned}
$$

Since $\left|\Phi_{n+1}(0)\right|<1$, we obtain

$$
\left|\frac{\Phi_{n+1}^{*}(z)}{I_{p}^{*}(z)}-E_{q}^{*}(z)\right|<\left|E_{q}^{*}(z)\right| \quad \text { for }|z|=1 .
$$


Thus, by Rouché's theorem, $E_{q}^{*}(z)$ has the same number of zeros inside $T$ as $\Phi_{n+1}^{*}(z)$. In other words, $\Phi_{n+1}^{*}(z)$ has $q$ zeros inside $T$ and thus $\Phi_{n+1}(z)$ has $q$ zeros outside and $p+1$ inside $T$.

(ii) If $|z|=1$, from (4) we have

$$
\left|\frac{\Phi_{n+1}^{*}(z)-\overline{\Phi_{n+1}(0)} z \Phi_{n}(z)}{I_{p}^{*}(z)}\right|=\left|\frac{\Phi_{n}^{*}(z)}{I_{p}^{*}(z)}\right|=\left|\frac{\Phi_{n}(z)}{I_{p}^{*}(z)}\right| .
$$

Hence

$$
\left|\frac{\Phi_{n+1}^{*}(z)}{I_{p}^{*}(z)}-\frac{\overline{\Phi_{n+1}(0)} z \Phi_{n}(z)}{I_{p}^{*}(z)}\right|=\frac{1}{\left|\Phi_{n+1}(0)\right|}\left|\frac{\overline{\Phi_{n+1}(0)} z \Phi_{n}(z)}{I_{p}^{*}(z)}\right| .
$$

From $\left|\Phi_{n+1}(0)\right|>1$ we get

$$
\left|\frac{\Phi_{n+1}^{*}(z)}{I_{p}^{*}(z)}-\frac{\overline{\Phi_{n+1}(0)} z \Phi_{n}(z)}{I_{p}^{*}(z)}\right|<\left|\frac{\overline{\Phi_{n+1}(0)} z \Phi_{n}(z)}{I_{p}^{*}(z)}\right| .
$$

Again by Rouché's theorem, $\Phi_{n+1}^{*}(z)$ and $z \Phi_{n}(z)$ have the same number of zeros inside $T$. Thus, $\Phi_{n+1}(z)$ has $q$ zeros inside and $p+1$ zeros outside $T$.

As a corollary of Proposition 3.4 we have the following result due to M. G. Krein.

COROLLARY 3.5. Let $\alpha_{n}$ be the number of permanences of sign in the sequence $1, \Delta_{0}, \Delta_{1}, \ldots, \Delta_{n-1}$ and $\beta_{n}$ the number of changes of sign in the same sequence. If for $n \geq 1, \Delta_{n} \neq 0$, then

(i) If $\Delta_{n} \Delta_{n-1}>0$, then $\Phi_{n}(z)$ has $\alpha_{n}$ zeros inside $T$ (counted with multiplicities).

(ii) If $\Delta_{n} \Delta_{n-1}<0$, then $\Phi_{n}(z)$ has $\beta_{n}$ zeros inside $T$ (counted with multiplicities).

From this, we conclude that the number of zeros of $\Phi_{n}(z)$ inside $T$ is equal to the number of positive eigenvalues of the moment matrix defining $\Phi_{n}(z)$. In fact, the signature (number of positive eigenvalues) of an Hermitian matrix such that $\Delta_{k} \neq 0$ for $k=0, \ldots, n$ is given by the number of positive terms of the sequence $1, \Delta_{0}, \Delta_{1} / \Delta_{0}, \ldots, \Delta_{n} / \Delta_{n-1}$.

Re marks. On the other hand, notice that given $p(z)=\sum_{k=0}^{n} a_{k} z^{k}$ and $a_{n} \neq 0$, it was shown in $[8]$ that $p(z)$ is orthogonal to $\mathbb{P}_{n-1}$ if the following three conditions hold:

(i) $a_{n} \in \mathbb{R}$.

(ii) $p(z)$ has no zeros on the unit circle.

(iii) If $p(\alpha)=0$ then $p\left(\overline{\alpha^{-1}}\right) \neq 0$.

But we cannot guarantee that $p(z)$ belongs to a sequence of orthogonal 
polynomials like the ones defined in 2.1. In fact, if we consider the Toeplitz matrix

$$
\left[\begin{array}{lll}
0 & 1 & 1 \\
1 & 0 & 1 \\
1 & 1 & 0
\end{array}\right]
$$

then $\Phi_{2}(z)=z^{2}-z-1$ does not belong to a sequence of orthogonal polynomials because $\left|\Phi_{2}(0)\right|=1$.

In the case of orthogonal polynomials with respect to positive definite Hankel matrices, it is known that such polynomials are the characteristic polynomials of the principal submatrix of a tridiagonal symmetric (Jacobi) matrix. The elements of such a matrix are related to the coefficients of the three-term recurrence relation satisfied by the polynomials.

For the orthogonal polynomials associated with a Toeplitz matrix it can be proved (see [6]) that these polynomials are also the characteristic polynomials of the principal submatrices of a certain Hessenberg matrix, whose elements are related to the Szegö parameters.

\section{References}

[1] R. L. Ellis, I. Gohberg and D. C. Lay, On two theorems of M. G. Krein concerning polynomials orthogonal on the unit circle, Integral Equations Operator Theory 11 (1988), 87-103.

[2] T. Erdelyi, P. Nevai, J. Zhang and J. S. Geronimo, Simple proof of Favard's theorem on the unit circle, Atti Sem. Mat. Fis. Univ. Modena 29 (1991), 41-46.

[3] G. Freud, Orthogonal Polynomials, Pergamon Press, Oxford, 1971.

[4] P. García Lázaro, Polinomios ortogonales y distribuciones, Doctoral Dissertation, Publ. Sem. Mat. García de Galdeano, Ser. II, 32, Universidad de Zaragoza, 1990.

[5] Y. L. Geronimus, Polynomials orthogonal on a circle and their applications, Amer. Math. Soc. Transl. Ser. 1, 3 (1962), 1-78.

[6] E. Godoy and F. Marcellán, Orthogonal polynomials on the unit circle: Distribution of zeros, J. Comput. Appl. Math. 37 (1991), 265-272.

[7] W. B. Jones, O. Njastad and W. J. Thron, Moment theory, orthogonal polynomials, quadrature, and continued fractions associated with the unit circle, Bull. London Math. Soc. 21 (1989), 113-152.

[8] M. G. Krein, On the distribution of the roots of polynomials which are orthogonal on the unit circle with respect to an alternating weight, Teor. Funktsiŭ Funktsional. Anal. i Prilozhen. 2 (1966), 131-137.

[9] H. J. Landau, Polynomials orthogonal in an indefinite metric, in: Orthogonal Matrix-Valued Polynomials and Applications, I. Gohberg (ed.), Birkhäuser, Basel, 1988, 203-214.

[10] F. Marcellán, Orthogonal polynomials and Toeplitz matrices: Some applications, in: Rational Approximation and Orthogonal Polynomials, M. Alfaro (ed.), Publ. Sem. Mat. García de Galdeano, Zaragoza, 1989, 31-57. 
[11] F. Marcellán and M. Alfaro, Recent trends in orthogonal polynomials on the unit circle, in: Orthogonal Polynomials and their Applications, C. Brezinski et al. (eds.), IMACS Ann. Comput. Appl. Math. 9 (1991), 3-14.

[12] G. Szegö, Orthogonal Polynomials, 4th ed., Amer. Math. Soc. Colloq. Publ. 23, Providence, 1975.

P. García Lázaro

DEPARTAMENTO DE MATEMÁTICA APLICADA E.T.S.I. INDUSTRIALES

UNIVERSIDAD POLITÉCNICA

C/ JOSÉ GUTIERREZ ABASCAL, 2

28006 MADRID, SPAIN
F. Marcellán

DEPARTAMENTO DE INGENIERIA ESCUELA POLITÉCNICA SUPERIOR UNIVERSIDAD CARLOS III DE MADRID AVENIDA MEDITERRANEO 20 28913 LEGANES (MADRID), SPAIN

Reçu par la Rédaction le 28.8.1992

Révisé le 14.6.1993 